\title{
Hospitalization and cost after switching from atypical to typical antipsychotics in schizophrenia patients in Thailand
}

This article was published in the following Dove Press journal:

ClinicoEconomics and Outcomes Research

29 April 2016

Number of times this article has been viewed

\section{Tuanthon Boonlue ${ }^{1,2}$ \\ Suphat Subongkot ${ }^{1,2}$ \\ Piyameth Dilokthornsakul ${ }^{3,4}$ \\ Ronnachai Kongsakon ${ }^{5}$ \\ Oraluck Pattanaprateep 6 \\ Orabhorn Suanchang ${ }^{7}$ \\ Nathorn Chaiyakunapruk ${ }^{3,8-10}$}

'Clinical Pharmacy Division, Faculty of Pharmaceutical Sciences, Khon

Kaen University, Khon Kaen, Thailand;

${ }^{2}$ The College of Pharmacotherapy of Thailand, Nonthaburi, Thailand; ${ }^{3}$ Center of Pharmaceutical Outcomes Research, Department of Pharmacy Practice, Faculty of Pharmaceutical Sciences, Naresuan University, Phitsanulok, Thailand; ${ }^{4}$ Center for Pharmaceutical Outcomes Research, University of Colorado Skaggs School of Pharmacy and Pharmaceutical Sciences, Aurora, CO, USA;

${ }^{5}$ Department of Psychiatry, Faculty of Medicine, Ramathibodi Hospital, Mahidol University, Bangkok, Thailand; ${ }^{6}$ Department of Health Informatics, Ramathibodi Hospital, Mahidol University, ${ }^{7}$ Department of Pharmacy, Somdet Chaopraya Institute of Psychiatry, Bangkok, Thailand; ${ }^{8}$ School of Pharmacy, Monash University Malaysia, Selangor, Malaysia; ' 5 Shool of Population Health, University of Queensland, Brisbane, Australia; ${ }^{10}$ School of Pharmacy, University of Wisconsin-Madison, Madison, WI, USA

Correspondence: Nathorn Chaiyakunapruk School of Pharmacy, Monash University Malaysia, Jalan Lagoon Selatan,

46150 Bandar Sunway, Selangor, Malaysia

Tel +60355144413

Fax +60355146326

Email nathorn.chaiyakunapruk@monash. edu
Background: Several clinical practice guidelines suggest using atypical over typical antipsychotics in patients diagnosed with schizophrenia. Nevertheless, cost-containment policy urged restricting usage of atypical antipsychotics and switching from atypical to typical antipsychotics.

Objective: This study aimed to evaluate clinical and economic impacts of switching from atypical to typical antipsychotics in schizophrenia patients in Thailand.

Methods: From October 2010 through September 2013, a retrospective cohort study was performed utilizing electronic database of two tertiary hospitals. Schizophrenia patients aged 18 years or older and being treated with atypical antipsychotics were included. Patients were classified as atypical antipsychotic switching group if they switched to typical antipsychotics after 180 days of continual atypical antipsychotics therapy. Outcomes were schizophrenia-related hospitalization and total health care cost. Logistic and Poisson regression were used to evaluate the risk of hospitalization, and generalized linear model with gamma distribution was used to determine the health care cost. All analyses were adjusted by employing propensity score and multivariable analyses. All cost estimates were adjusted according to 2013 consumer price index and converted to US\$ at an exchange rate of 32.85 Thai bahts/US\$.

Results: A total of 2,354 patients were included. Of them, 166 (7.1\%) patients switched to typical antipsychotics. The adjusted odds ratio for schizophrenia-related hospitalization in atypical antipsychotic switching group was 1.87 (95\% confidence interval [CI] 1.23-2.83). The adjusted incidence rate ratio was 2.44 (95\% CI 1.57-3.79) for schizophrenia-related hospitalizations. The average total health care cost was lower in patients with antipsychotic switching $(-\$ 64$; $95 \%$ CI $-\$ 459$ to $\$ 332$ ).

Conclusion: Switching from atypical to typical antipsychotics is associated with an increased risk of schizophrenia-related hospitalization. Nonetheless, association with average total health care cost was not observed. These findings can be of use as a part of evidence in executing prospective cost-containment policy.

Keywords: antipsychotic switching, schizophrenia, hospitalization, cost, atypical antipsychotics, typical antipsychotics

\section{Introduction}

Schizophrenia is one of the most debilitating mental disorders with high disability rate. ${ }^{1}$ The global prevalence of schizophrenia is $\sim 1 \%{ }^{2}$ Since 2004 , it has been ranked as the fifth and sixth cause of health-related morbidity among males and females, respectively, worldwide. ${ }^{3}$ Schizophrenia is significantly associated with humanistic ${ }^{4}$ and economic burdens internationally, especially in Asia. ${ }^{5,6}$ 
Antipsychotics are mainstay for management of patients diagnosed with schizophrenia. ${ }^{7}$ American Psychiatric Association Practice Guidelines $2004^{8}$ and its 2009 update, ${ }^{9}$ the 2005 International Psychopharmacology Algorithm Project, ${ }^{10}$ and the 2006 Texas Medication Algorithm Project ${ }^{11}$ have recommended atypical antipsychotics as drugs of choice given that they exert an optimal efficacy ${ }^{12,13}$ and improved safety/ tolerability profile, ${ }^{14}$ compared to those of typical antipsychotics. In developing countries such as Thailand, national clinical practice guideline allows the usage of both typical and atypical antipsychotics as recommended therapy. ${ }^{15}$ As of currently, all typical (eg, haloperidol, chlorpromazine) and only some atypical (eg, risperidone, clozapine) antipsychotics have been included in the National List of Essential Medicines, ${ }^{16}$ which is a standard coverage for beneficiaries in all main public health sectors in Thailand.

Due to the increasing health expenditures and budget constraints, cost-containment strategies have been implemented in most developed ${ }^{17}$ and developing countries ${ }^{18}$ to efficiently ensure expenditure control. The advocacy to use typical antipsychotics for new cases as well as switching of atypical to typical antipsychotics has been proposed as a potential cost-containment policy for schizophrenia management in Thailand. Still, clinicians and professional health care organizations voiced their concerns regarding the potential implications of such policy. Therefore, there is an urgent need to understand potential impacts of the antipsychotic switching policy on both clinical and economic aspects. This study aims to determine the effects of switching from atypical to typical antipsychotics on hospitalization and health care cost in Thailand. We hypothesized that schizophrenia patients who switched from atypical to typical antipsychotics had higher risk for hospitalization and higher costs than those who continued on atypical antipsychotics.

\section{Methods}

\section{Setting and data sources}

A retrospective cohort study using electronic databases from university-based hospital and a tertiary psychiatric hospital in Bangkok, Thailand, was undertaken. The inpatient and outpatient database, and pharmacy and financial database were analyzed. Patients' demographic characteristics (eg, age, sex, insurance) and diagnostics code (International Classification of Disease version 10 [ICD-10]) were extracted from inpatient and outpatient databases. In Thailand, health coverage schemes ${ }^{19}$ consisted of the Civil Servant Medical Benefit Scheme for government employees and state enterprise employees, the Social Security Scheme and Workman's Compensation Fund for private sector employees, the Universal Coverage Scheme and fee exemption for the poor and underprivileged, and others such as private insurance and out-of-pocket payments for patients who are uninsured. Information on drug's name, amount of drug prescribed, and treatment regimen were extracted from pharmacy database from October 2010 to September 2013. Information on date of payment, type of payment, and medical charges (medication, medical service, laboratory charge, and other

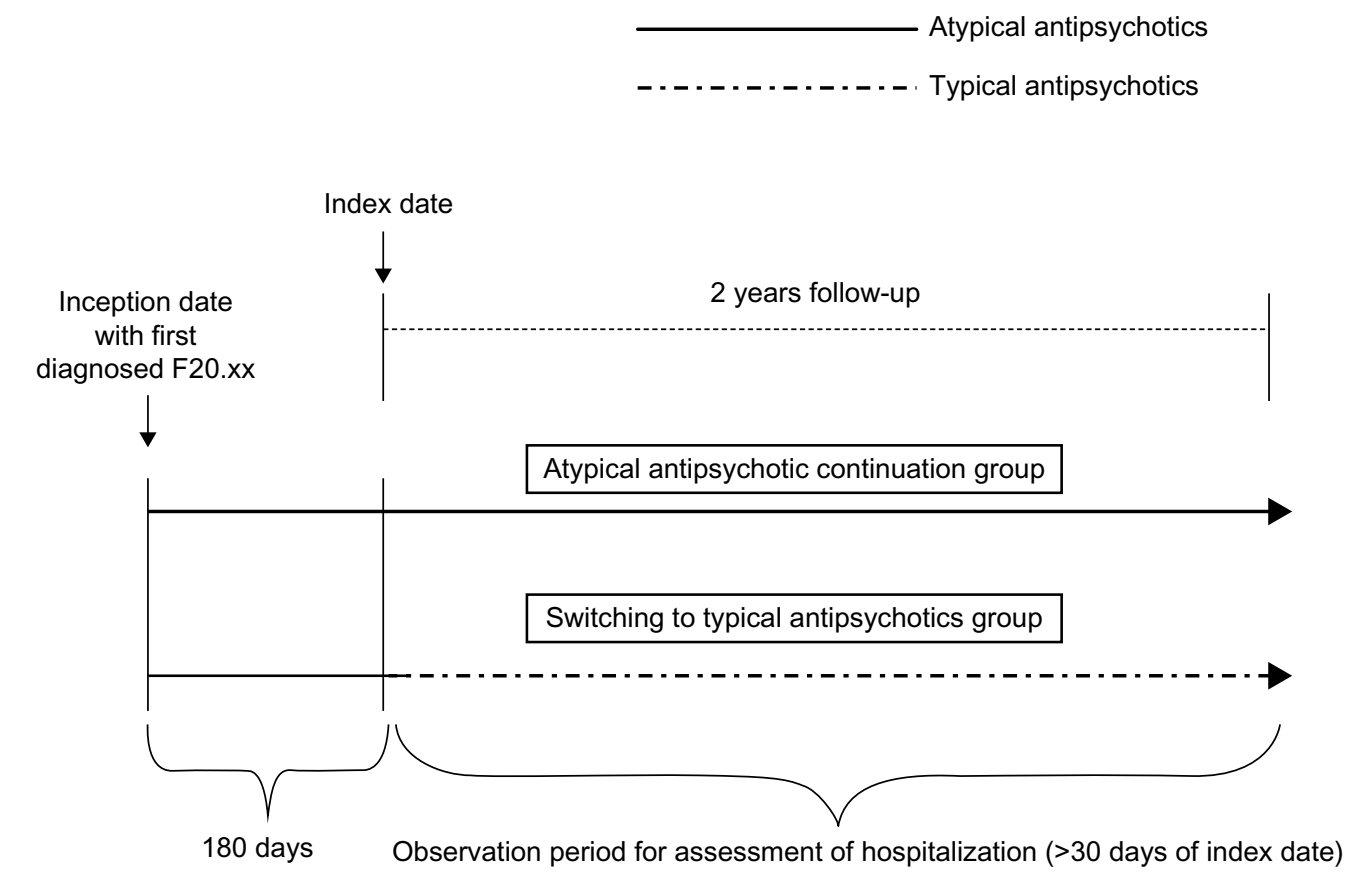

Figure I Selection of cohort for evaluation of hospitalization and duration. 
charges) were extracted from financial database. The study protocol was submitted to, and approved by, the Ethical Review Committees of both Ramathibodi Hospital and Somdet Choapraya Institute of Psychiatry in accordance with the Declaration of Helsinki. As this study is retrospective, the Ethical Review Committees deemed written informed consent is not required.

\section{Patient selection and study period}

Patients who visited outpatient departments in one of the university hospitals and one of the tertiary psychiatric hospitals in Bangkok metropolitan area and met the following inclusion criteria were included in the study: 1) diagnosed as schizophrenia (defined by ICD-10: F20.xx) during the period from October 2010 through April 2011;2) aged 18 years or older; 3) received atypical antipsychotics (clozapine, olanzapine, quetiapine, aripiprazole, risperidone, paliperidone) at least 180 days before the date of switching to typical antipsychotics (index date) or not switching to typical antipsychotics or other atypical antipsychotics. Typical antipsychotics - both oral and parenteral dosage forms - comprise haloperidol, thioridazine, trifluoperazine, fluphenazine, perphenazine, zuclopenthixol, flupenthixol, and pimozide. Patients receiving a combination of atypical and typical antipsychotics during our study period were excluded. Patient data during the period of 180 days prior to the index date were used to determine propensity score and follow outcomes of interest of adult schizophrenia patients up to 2 years. Patients were categorized into two cohorts as follows: 1) atypical antipsychotic switching to typical antipsychotics group and 2) atypical antipsychotic continuation group by the index date defined as the date of switching from atypical to typical antipsychotics in the former cohort and the first date after continuation of atypical antipsychotics for 180 days in the latter cohort. The selection of cohort was shown in Figure 1.

\section{Outcomes of interest}

Schizophrenia-related hospitalization according to ICD-10 (F20.xx) was selected as a primary outcome. The secondary outcomes were all-cause hospitalization, health care resource utilization, and related health care costs. Allcause hospitalization was described as any hospitalization that can be categorized by ICD-10 during the 2-year study period. Health care resource utilizations were measured in terms of number of outpatient visits, number of inpatient visits, and length of hospitalization. Total health care cost included outpatient- and inpatient-related costs and was not limited to the medication costs. Both total health care cost and medication costs were calculated from charges using a cost-to-charge ratio among Thailand University hospital which appeared to be $0.73 .^{20}$

\section{Statistical analysis}

The baseline sociodemographic and clinical characteristics of patients were summarized using descriptive statistics and were compared using chi-square or Fisher's exact tests (for categorical variables) or nonparametric Wilcoxon rank-sum test (for numerical variables).

Primary analyses were conducted on patients in atypical antipsychotic continuation group and compared with atypical antipsychotic switching group. We performed sensitivity analyses on patients switching from atypical antipsychotics to other atypical antipsychotics to explore the consistency of the effect of atypical antipsychotic drugs class.

A multivariate logistic regression was used to determine associations of antipsychotic switching with schizophreniarelated and all-cause hospitalizations with the use of conditional logistic regression models for matched analysis, while a multivariate Poisson regression was used to determine associations of antipsychotic switching with schizophreniarelated and all-cause hospitalization rates. A multivariate generalized linear model with log-link function and gamma distribution was used to determine total health care cost, pharmacy cost, outpatient cost, and inpatient cost. The health care cost was converted to 2013 value using consumer price inde ${ }^{21}$ and was subsequently converted to US\$ according to an exchange rate of 32.85 Thai bahts/US\$. ${ }^{22}$ Propensity score (PS) was calculated and used to adjust baseline characteristics imbalance between the two groups. For this purpose, logistic regression model was initially developed to estimate a propensity score for each patient based on baseline covariates including age, sex, health insurance, occupation, Charlson comorbidity index score, number of previous hospitalizations, and concomitant psychotropic medications (anticholinergics, antidepressants, mood stabilizers, and benzodiazepines). The propensity score was later applied as a matching variable to pair patients who switched antipsychotics to patients who continued on atypical antipsychotics using 1:1 nearest neighbor matching technique. The propensity score was also applied as a covariate in different analyses. Statistical analysis was performed using STATA version 12 . The statistical significant level was set at $P<0.05$ in a two-sided test.

\section{Results \\ Patient characteristics}

As shown in Figure 2, a total of 20,910 patients were identified; however, only 2,354 patients met our inclusion criteria. Applying a propensity score matching technique, the number 


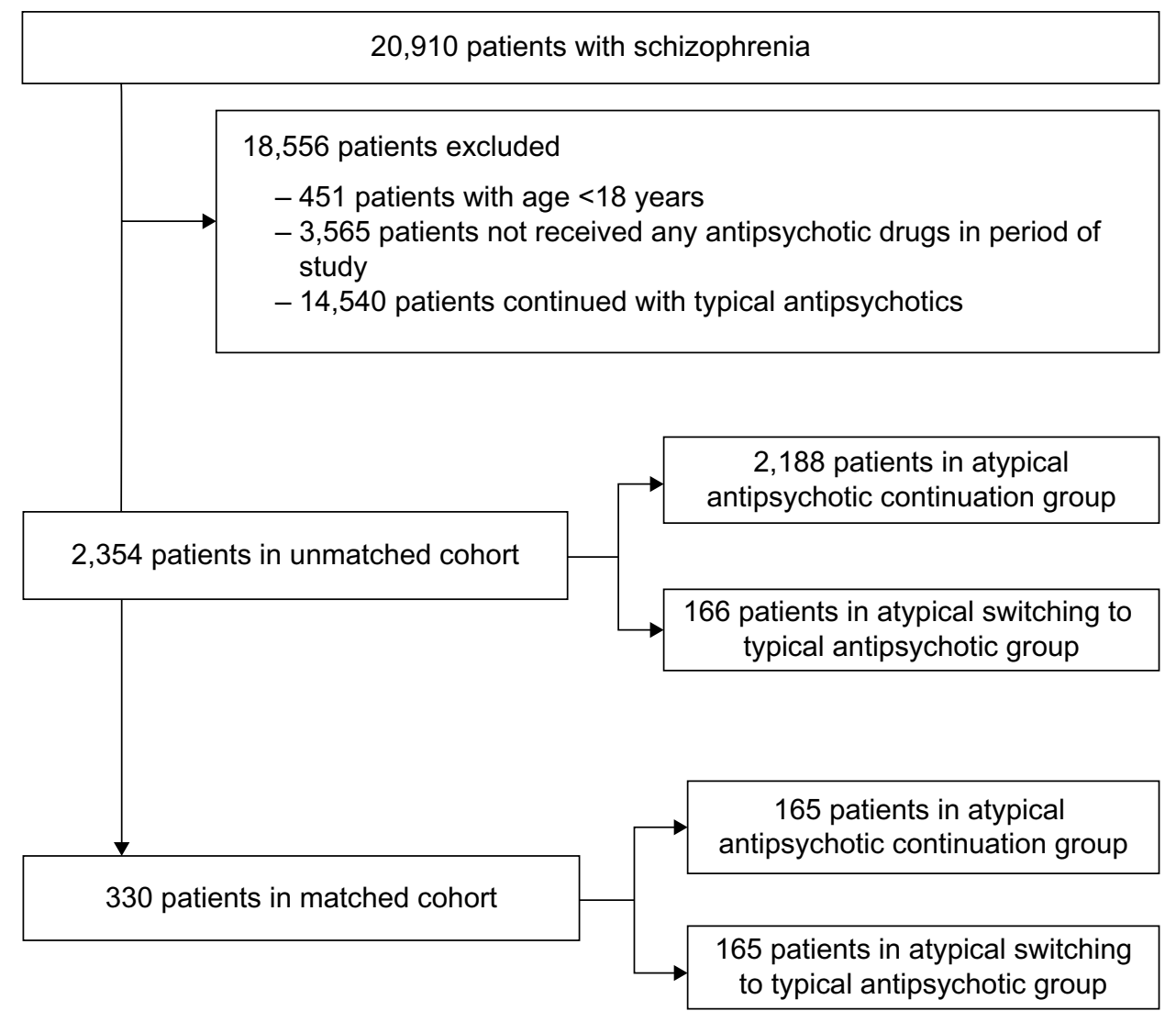

Figure 2 A study flow chart.

of patients included in a matched cohort was 330 patients (165 patients for each group).

Patient demographics are shown in Table 1. The unmatched analysis of two groups yielded significant differences in age with a higher mean in atypical antipsychotics continuation, the average age was $42.8 \pm 13.6$ years. Percentages of male were similar in both the groups. For matched cohort, there were no significant differences in any baseline characteristics between the two groups except for occupation.

\section{Effects of antipsychotic switching on schizophrenia-related and all-cause hospitalizations}

For unmatched cohort, the unadjusted odds ratios (OR) for schizophrenia-related and all-cause hospitalizations within 2-year follow-up between antipsychotic switching and antipsychotic continuation groups were 2.49 (95\% CI 1.70-3.66) and 2.25 (95\% CI 1.54-3.29), respectively. Based on a multivariable logistic regression analysis, the adjusted OR was 1.87 (95\% CI 1.23-2.83) and 1.77 (95\% CI, 1.17-2.68), respectively. Based on the PS-matched cohort, all observed hospitalizations were schizophrenia-related hospitalizations. The adjusted OR for schizophrenia-related hospitalizations within 2-year follow-up period was 4.62 (95\% CI 0.99-21.62). In sensitivity analyses using atypical antipsychotic switching to other atypical antipsychotics as comparator, the outcomes result was similar to that obtained for primary analyses (Table 2).

Based on multivariable Poisson regression analyses within unmatched cohort, the PS-adjusted incidence rate ratios for schizophrenia hospitalization and all-cause hospitalizations within 2 years were 2.44 (95\% CI 1.57-3.79) and 2.89 (95\% CI 2.16-3.86), respectively. For PS-matched cohort, the adjusted incidence rate ratios for schizophrenia hospitalization and all-cause hospitalizations were 2.18 (95\% CI 1.34-3.56) and 2.34 (95\% CI 1.66-3.31), respectively. The results of the sensitivity analyses also show similar incidence rate ratios (Table 3).

\section{Resource utilization during the follow-up period}

The number of outpatient visits, inpatient visits, and length of stays were significantly higher in atypical antipsychotic switching group for unmatched cohort. However, in matched 
Table I Baseline characteristics

\begin{tabular}{|c|c|c|c|c|c|c|}
\hline \multirow[t]{2}{*}{ Characteristics } & \multicolumn{3}{|c|}{ Unmatched cohort $(\mathrm{N}=2,354)$} & \multicolumn{3}{|c|}{ Matched cohort $(\mathrm{N}=330)$} \\
\hline & $\begin{array}{l}\text { Atypical } \\
\text { antipsychotic } \\
\text { continuation } \\
(\mathbf{N}=\mathbf{2 , 1 8 8 )}\end{array}$ & $\begin{array}{l}\text { Atypical } \\
\text { antipsychotic } \\
\text { switching } \\
(\mathrm{N}=166)\end{array}$ & $P$-value & $\begin{array}{l}\text { Atypical } \\
\text { antipsychotic } \\
\text { continuation } \\
(\mathrm{N}=165)\end{array}$ & $\begin{array}{l}\text { Atypical } \\
\text { antipsychotic } \\
\text { switching } \\
(\mathrm{N}=165)\end{array}$ & $P$-value \\
\hline \multicolumn{7}{|c|}{ Demographic variables } \\
\hline Age, mean (SD), years & $42.8(13.6)$ & $39.2(13.4)$ & 0.001 & 39.1 (II.9) & $39.3(13.4)$ & 0.85 \\
\hline \multicolumn{7}{|l|}{ Sex } \\
\hline Female, n (\%) & I, I I $3(50.9)$ & $77(46.4)$ & 0.27 & $75(45.5)$ & $77(46.7)$ & 0.83 \\
\hline \multicolumn{7}{|c|}{ Socioeconomic variables, N (\%) } \\
\hline \multicolumn{7}{|l|}{ Marital status } \\
\hline Married & $944(43.1)$ & $75(45.2)$ & 0.61 & $73(44.2)$ & $74(44.9)$ & 0.91 \\
\hline Unmarried & $\mathrm{I}, 244(56.9)$ & $91(54.8)$ & & $92(55.8)$ & $91(55.1)$ & \\
\hline \multicolumn{7}{|l|}{ Religion } \\
\hline Buddhist & $2,124(97.1)$ & $162(97.6)$ & 0.70 & $162(98.2)$ & $161(97.6)$ & 0.70 \\
\hline Christian & $27(1.2)$ & $3(1.8)$ & 0.53 & $2(1.2)$ & $3(1.8)$ & 0.65 \\
\hline Muslim & $37(1.7)$ & $\mathrm{I}(0.6)$ & 0.28 & $\mathrm{I}(0.6)$ & $\mathrm{I}(0.6)$ & 1.00 \\
\hline \multicolumn{7}{|l|}{ Occupation } \\
\hline Government officer & $138(6.3)$ & $8(4.8)$ & 0.44 & $4(2.5)$ & $8(5.0)$ & 0.24 \\
\hline Housewife & $555(25.4)$ & $30(18.1)$ & 0.04 & $43(26.1)$ & $30(18.2)$ & 0.09 \\
\hline Self-employed & $139(6.4)$ & II (6.6) & 0.89 & II (6.7) & II (6.7) & 1.00 \\
\hline Student & $467(2 \mid .3)$ & $55(33.1)$ & $<0.001$ & $36(21.8)$ & $54(32.7)$ & 0.03 \\
\hline Blue collar & $149(6.8)$ & $13(7.8)$ & 0.62 & $13(7.9)$ & $13(7.9)$ & 1.00 \\
\hline Farmer & $9(0.4)$ & $2(1.2)$ & 0.15 & 0 & $2(1.2)$ & 0.16 \\
\hline Unemployed & $8(0.4)$ & 0 & 0.44 & 0 & 0 & $N / A$ \\
\hline Unknown & $723(33.0)$ & $47(28.3)$ & 0.21 & $58(35.0)$ & $47(28.5)$ & 0.19 \\
\hline \multicolumn{7}{|l|}{ Health coverage } \\
\hline UC & $\mathrm{I},|3|(5 \mid .7)$ & $109(65.7)$ & 0.001 & $116(68.3)$ & $108(64.6)$ & 0.35 \\
\hline SSS & $112(5.1)$ & $8(4.8)$ & 0.87 & $10(6.1)$ & $8(4.9)$ & 0.48 \\
\hline CSMBS & $285(13.0)$ & $17(10.2)$ & 0.30 & II (6.7) & $17(10.3)$ & 0.24 \\
\hline Others & $660(30.2)$ & $32(19.3)$ & 0.003 & $28(17.0)$ & $32(19.4)$ & 0.57 \\
\hline \multicolumn{7}{|l|}{ Concomitant medications } \\
\hline Antidepressants & $779(35.6)$ & $65(39.2)$ & 0.36 & $62(37.6)$ & $65(39.4)$ & 0.73 \\
\hline Mood stabilizers & $506(23.1)$ & $53(31.9)$ & 0.01 & $46(27.9)$ & $52(31.5)$ & 0.47 \\
\hline Benzodiazepines & I,304 (59.6) & $143(86.1)$ & $<0.001$ & I4I (85.5) & I42 (86.I) & 0.88 \\
\hline Anticholinergics & $\mathrm{I}, 48 \mathrm{I}(67.7)$ & $149(89.8)$ & $<0.001$ & I47 (89.1) & I 48 (89.7) & 0.86 \\
\hline \multicolumn{7}{|l|}{ Charlson index score } \\
\hline 0 & I,93I (88.3) & I $45(87.4)$ & 0.73 & $145(88.2)$ & $145(88.2)$ & 1.00 \\
\hline I & $178(8.1)$ & $18(10.8)$ & 0.22 & $18(10.9)$ & $17(10.3)$ & 0.86 \\
\hline$\geq 2$ & $79(3.6)$ & $3(1.8)$ & 0.22 & $2(1.2)$ & $3(1.8)$ & 0.65 \\
\hline \multicolumn{7}{|c|}{ Number of previous hospitalizations ${ }^{a}$} \\
\hline 0 & $2,028(92.7)$ & I $56(94.0)$ & 0.54 & $157(95.2)$ & $156(94.6)$ & 0.80 \\
\hline 1 & $143(6.5)$ & $7(4.2)$ & 0.24 & $6(3.6)$ & $7(4.2)$ & 0.78 \\
\hline$\geq 2$ & $17(0.8)$ & $3(1.8)$ & 0.07 & $2(1.2)$ & $2(1.2)$ & 1.00 \\
\hline
\end{tabular}

Note: ${ }^{a}$ Number of hospitalization in inclusion period (180 days).

Abbreviations: UC, Universal Coverage Scheme; SSS, Social Security Scheme; CSMBS, Civil Servant Medical Benefit scheme; NA, not available.

cohort, there was no significant difference between atypical antipsychotic switching and continuation groups (Table 4).

\section{Health care cost utilization during the follow-up period}

The adjusted differences in total health care cost for unmatched and matched cohort were - \$64 (95\% CI - \$459 to $\$ 332)$ and $-\$ 222(95 \% \mathrm{CI}-\$ 713$ to $\$ 268)$, respectively.
When inpatient and outpatient costs were analyzed separately, there were no significant differences between the antipsychotic switching and continuation groups. In sensitivity analyses, outpatients health care cost in atypical antipsychotic switching to typical antipsychotics was lower than that observed for atypical antipsychotic switching to other atypical antipsychotics in unmatched cohort with a difference of $\$ 1,226(\$ 510-\$ 1,942)$ for total outpatient cost and \$1,208 
Table 2 Logistic regression analysis of hospitalization between atypical antipsychotic switching to typical antipsychotics and atypical antipsychotic continuation groups

\begin{tabular}{|c|c|c|c|c|}
\hline \multirow[t]{2}{*}{ Outcomes measure } & \multicolumn{2}{|l|}{ Unadjusted model } & \multicolumn{2}{|l|}{ Adjusted model ${ }^{\mathrm{a}}$} \\
\hline & Odds ratio $(95 \% \mathrm{Cl})$ & $P$-value & Odds ratio $(95 \% \mathrm{Cl})$ & $P$-value \\
\hline \multicolumn{5}{|c|}{ Atypical antipsychotic switching to typical antipsychotics vs atypical antipsychotic continuation: unmatched cohort $(\mathrm{N}=2,354)$} \\
\hline Schizophrenia-related hospitalization & $3.13(2.11-4.63)$ & $<0.001$ & $2.23(1.45-3.45)$ & $<0.001$ \\
\hline All-cause hospitalization & $2.72(1.84-4.01)$ & $<0.001$ & $1.98(1.29-3.06)$ & 0.002 \\
\hline \multicolumn{5}{|c|}{ Atypical antipsychotic switching to typical antipsychotics vs atypical antipsychotic continuation: matched cohort ( $\mathrm{N}=330)$} \\
\hline Schizophrenia-related hospitalization & $4.28(0.92-19.85)$ & 0.06 & $4.28(0.92-19.85)$ & 0.06 \\
\hline All-cause hospitalization & $4.28(0.92-19.85)$ & 0.06 & $4.28(0.92-19.85)$ & 0.06 \\
\hline \multicolumn{5}{|c|}{ Atypical antipsychotic switching to typical antipsychotics vs atypical antipsychotic switching to other atypical antipsychotics: unmatched cohort ( $\mathrm{N}=452$ ) } \\
\hline Schizophrenia-related hospitalization & $1.64(1.02-2.66)$ & 0.04 & $2.35(1.34-4.11)$ & 0.01 \\
\hline All-cause hospitalization & $1.49(0.93-2.38)$ & $<0.001$ & $2.00(1.16-3.48)$ & 0.007 \\
\hline \multicolumn{5}{|c|}{ Atypical antipsychotic switching to typical antipsychotics vs atypical antipsychotic switching to other atypical antipsychotics: matched cohort ( $\mathrm{N}=3 \mid 0)$} \\
\hline Schizophrenia-related hospitalization & $2.58(0.26-25.20)$ & 0.42 & $2.58(0.26-25.20)$ & 0.42 \\
\hline All-cause hospitalization & $2.58(0.26-25.20)$ & 0.42 & $2.58(0.26-25.20)$ & 0.42 \\
\hline
\end{tabular}

Notes: ${ }^{a}$ Adjusted for age, sex, marital status, religion, health coverage, concomitant medication, Charlson index score, propensity score.

Abbreviation: $\mathrm{Cl}$, confidence interval.

Table 3 Poisson regression analysis between atypical antipsychotic switching to typical antipsychotics and atypical antipsychotic continuation groups

\begin{tabular}{|c|c|c|c|c|}
\hline \multirow[t]{2}{*}{ Outcomes measure } & \multicolumn{2}{|l|}{ Unadjusted model } & \multicolumn{2}{|l|}{ Adjusted model ${ }^{\mathrm{a}}$} \\
\hline & $\begin{array}{l}\text { Incidence rate ratio } \\
(95 \% \mathrm{Cl})\end{array}$ & P-value & $\begin{array}{l}\text { Incidence rate ratio } \\
(95 \% \mathrm{Cl})\end{array}$ & P-value \\
\hline \multicolumn{5}{|c|}{ Atypical antipsychotic switching to typical antipsychotics vs atypical antipsychotic continuation: unmatched cohort $(\mathrm{N}=2,354)$} \\
\hline Schizophrenia-related hospitalization & $4.08(2.9 \mid-5.7 I)$ & $<0.001$ & $2.56(1.79-3.66)$ & $<0.001$ \\
\hline All-cause hospitalization & $3.79(2.86-5.04)$ & $<0.001$ & $2.96(2.19-3.99)$ & $<0.001$ \\
\hline \multicolumn{5}{|c|}{ Atypical antipsychotic switching to typical antipsychotics vs atypical antipsychotic continuation: matched cohort ( $N=330$ ) } \\
\hline Schizophrenia-related hospitalization & $2.50(1.54-4.06)$ & $<0.001$ & $3.18(1.84-5.50)$ & $<0.001$ \\
\hline All-cause hospitalization & $2.46(1.64-3.72)$ & $<0.001$ & $2.73(I .73-4.3 I)$ & $<0.001$ \\
\hline \multicolumn{5}{|c|}{$\begin{array}{l}\text { Atypical antipsychotic switching to typical antipsychotics vs atypical antipsychotic switching to other atypical antipsychotics: unmatched cohort } \\
(\mathrm{N}=452)\end{array}$} \\
\hline Schizophrenia-related hospitalization & $2.61(1.72-3.96)$ & $<0.001$ & $3.21(2.03-5.09)$ & $<0.001$ \\
\hline All-cause hospitalization & $3.01(2.09-4.33)$ & $<0.00$ I & $3.74(2.52-5.58)$ & $<0.001$ \\
\hline \multicolumn{5}{|c|}{ Atypical antipsychotic switching to typical antipsychotics vs atypical antipsychotic switching to other atypical antipsychotics: matched cohort ( $\mathrm{N}=310)$} \\
\hline Schizophrenia-related hospitalization & $3.09(1.82-5.27)$ & $<0.001$ & $3.39(1.94-5.90)$ & $<0.001$ \\
\hline All-cause hospitalization & $3.56(2.19-5.79)$ & $<0.001$ & $3.94(2.37-6.56)$ & $<0.001$ \\
\hline
\end{tabular}

Notes: ancidence rate ratio based on negative binomial or Poisson regression model, adjusted for time to switching and other covariates (sex, age, health coverage, previous hospitalization, Charlson index score, concomitant medication, propensity score).

Abbreviation: $\mathrm{Cl}$, confidence interval.

Table 4 Resource utilization of atypical antipsychotic continuation and switching to typical antipsychotics

\begin{tabular}{|c|c|c|c|c|}
\hline \multirow[t]{2}{*}{ Health resource utilization } & \multirow{2}{*}{$\frac{\text { AAP continuation }}{\text { Mean } \pm \text { SD }}$} & \multirow{2}{*}{$\frac{\text { AAP switching }}{\text { Mean } \pm \text { SD }}$} & \multirow[t]{2}{*}{ Difference } & \multirow[t]{2}{*}{$P$-value } \\
\hline & & & & \\
\hline \multicolumn{5}{|l|}{ Unmatched cohort $(\mathrm{N}=2,354)$} \\
\hline Outpatient visits (times) & $4.22 \pm 3.19$ & $5.07 \pm 5.27$ & $-0.85(-1.39$ to -0.31$)$ & 0.002 \\
\hline Inpatient visits (times) & $0.55 \pm 1.23$ & $1.32 \pm 1.83$ & $-0.77(-0.98$ to -0.57$)$ & $<0.001$ \\
\hline Length of stay (days) & $22.91 \pm 65.93$ & $62.66 \pm 124.58$ & $-39.75(-51.24$ to -28.26$)$ & $<0.001$ \\
\hline \multicolumn{5}{|l|}{ Matched cohort $(\mathrm{N}=330)$} \\
\hline Outpatient visits (times) & $4.52 \pm 3.73$ & $5.07 \pm 5.28$ & $-0.56(-1.54$ to 0.43$)$ & 0.27 \\
\hline Inpatient visits (times) & $0.89 \pm 1.73$ & $1.26 \pm 1.67$ & $-0.37(-0.74$ to -0.001$)$ & 0.05 \\
\hline Length of stay (days) & $33.39 \pm 79.27$ & $59.92 \pm 119.83$ & $-26.52(-48.53$ to -4.52$)$ & 0.02 \\
\hline
\end{tabular}

Abbreviations: SD, standard deviation; AAP, atypical antipsychotics. 
(\$541-\$1,875) for outpatient medication cost, but there was no significant difference for other outpatient costs and the results were similar to the matched cohort (Table 5).

\section{Discussion}

To our knowledge, this is the first study to determine the effects of antipsychotic switching from atypical to typical antipsychotics in schizophrenia patients on hospitalization and health care cost. The results demonstrated that patients who switched from atypical antipsychotics to typical antipsychotics had an increased risk of schizophrenia-related and all-cause hospitalizations than patients who continued taking atypical antipsychotics. However, total health care costs are similar between the two groups. These findings warrant policy makers and stakeholders to carefully implement the atypical to typical antipsychotic switching program.

Our study discovered higher rate of concomitant psychotropic drugs usage in atypical antipsychotic switching group

Table 5 Health care cost between atypical antipsychotic continuation and switching to typical antipsychotics

\begin{tabular}{|c|c|c|c|c|}
\hline \multirow[t]{2}{*}{ Health care cost ${ }^{a}$} & \multicolumn{2}{|l|}{ Unadjusted model } & \multicolumn{2}{|l|}{ Adjusted model ${ }^{b}$} \\
\hline & Difference & $P$-value & Difference & $P$-value \\
\hline \multicolumn{5}{|c|}{ Atypical antipsychotic continuation vs atypical antipsychotic switching to typical antipsychotics: unmatched cohort $(\mathrm{N}=2,354)$} \\
\hline Total direct medical cost & $26(-740$ to 793$)$ & 0.95 & $-64(-459$ to 332$)$ & 0.75 \\
\hline \multicolumn{5}{|l|}{ Outpatient health care cost } \\
\hline Total medical outpatient cost & -265 (-878 to 347$)$ & 0.40 & $-147(-519$ to 194$)$ & 0.40 \\
\hline Outpatient medication cost & $-379(-969$ to 210$)$ & 0.21 & $-200(-510$ to 108$)$ & 0.20 \\
\hline Other outpatient costs & $73(-23$ to 170$)$ & 0.14 & $28(-44$ to I0I) & 0.45 \\
\hline \multicolumn{5}{|l|}{ Inpatient health care cost } \\
\hline Total medical inpatient cost & 753 (330 to $I, 176)$ & $<0.001$ & $60 I(-490$ to $I, 692)$ & 0.28 \\
\hline Inpatient medication cost & $44(-40$ to 129$)$ & 0.31 & $38(-90$ to 166$)$ & 0.56 \\
\hline Other inpatient costs & 705 (336 to $I, 073)$ & $<0.001$ & $628(-363$ to 1,621$)$ & 0.21 \\
\hline \multicolumn{5}{|c|}{ Atypical antipsychotic continuation vs atypical antipsychotic switching to typical antipsychotics: matched cohort (N=330) } \\
\hline Total direct medical cost & $144(-602$ to 892$)$ & 0.70 & $-223(-7 \mid 3$ to 268$)$ & 0.37 \\
\hline \multicolumn{5}{|l|}{ Outpatient health care cost } \\
\hline Total medical outpatient cost & $-19(-662$ to 623$)$ & 0.95 & $-239(-652$ to 174$)$ & 0.26 \\
\hline Outpatient medication cost & $-59(-635$ to 516$)$ & 0.84 & $-72(-346$ to 201$)$ & 0.60 \\
\hline Other outpatient costs & $33(-219$ to 285$)$ & 0.80 & $5(-94$ to 105$)$ & 0.91 \\
\hline \multicolumn{5}{|l|}{ Inpatient health care cost } \\
\hline Total medical inpatient cost & $674(-5$ to 1,353$)$ & 0.052 & $536(-353$ to 1,425$)$ & 0.24 \\
\hline Inpatient medication cost & $49(-29$ to I26) & 0.22 & $-15(-90$ to 61$)$ & 0.71 \\
\hline Other inpatient costs & $625(-15$ to $I, 265)$ & 0.056 & $715(-259$ to 1,688$)$ & 0.15 \\
\hline \multicolumn{5}{|c|}{ Atypical antipsychotic switching within class vs atypical antipsychotic switching to typical antipsychotics: unmatched cohort ( $N=452)$} \\
\hline Total direct medical cost & $73(-493$ to 639$)$ & 0.80 & $192(-479$ to 864$)$ & 0.58 \\
\hline \multicolumn{5}{|l|}{ Outpatient health care cost } \\
\hline Total medical outpatient cost & $-948(-1,696$ to -199$)$ & 0.013 & $-1,226(-1,942$ to -510$)$ & 0.001 \\
\hline Outpatient medication cost & $-1,014(-1,734$ to -293$)$ & 0.006 & $-I, 208(-I, 875$ to $-54 I)$ & $<0.001$ \\
\hline Other outpatient costs & $40(-|6|$ to $24 \mid)$ & 0.70 & $-27(-165$ to 110$)$ & 0.70 \\
\hline \multicolumn{5}{|l|}{ Inpatient health care cost } \\
\hline Total medical inpatient cost & $33(-492$ to 558$)$ & 0.90 & $202(-83 \mid$ to $\mid 24)$ & 0.70 \\
\hline Inpatient medication cost & $-58(-167$ to 50$)$ & 0.29 & $-466(-1,803$ to 870$)$ & 0.49 \\
\hline Other inpatient costs & $84(-374$ to 542$)$ & 0.72 & $244(-876$ to 136$)$ & 0.67 \\
\hline \multicolumn{5}{|c|}{ Atypical antipsychotic switching within class vs atypical antipsychotic switching to typical antipsychotics: matched cohort $(\mathrm{N}=3 \mid 0)$} \\
\hline Total direct medical cost & 229 (-296 to 754$)$ & 0.39 & $183(-490$ to 857$)$ & 0.59 \\
\hline \multicolumn{5}{|l|}{ Outpatient health care cost } \\
\hline Total medical outpatient cost & $-702(-I, 527$ to $|2|)$ & 0.10 & $-1,133(-1,919$ to -347$)$ & 0.005 \\
\hline Outpatient medication cost & $-74 \mid(-1,519$ to 38$)$ & 0.06 & $-I,|2|(-1,987$ to -455$)$ & 0.002 \\
\hline Other outpatient costs & $33(-225$ to 291$)$ & 0.80 & $-6(-100$ to 88$)$ & 0.90 \\
\hline \multicolumn{5}{|l|}{ Inpatient health care cost } \\
\hline Total medical inpatient cost & $197(-255$ to 649$)$ & 0.39 & $258(-1,6 \mid 2$ to $2, \mid 29)$ & 0.79 \\
\hline Inpatient medication cost & $9(-45$ to 63$)$ & 0.74 & $86(-1,193$ to 1366$)$ & 0.89 \\
\hline Other inpatient costs & $188(-227$ to 602$)$ & 0.38 & $404(-251$ to 332$)$ & 0.79 \\
\hline
\end{tabular}

Notes: ${ }^{\$} \$ 1=32.85$ Thai Baht; badjusted for age, sex, marital status, health coverage, concomitant medication, Charlson index score, and number of previous hospitalizations. 
as compared to that of atypical antipsychotic continuation group. With reference to CATIE trial, the overall concomitant psychotropic drugs usage was found in $82 \%{ }^{23}$ of all treated patients. However, when redefined according to our study criteria by each agent - for instance, antidepressants - the proportion of use of antidepressants was found to be $\sim 38 \%$ in this study, but the proportion of use of other agents such as mood stabilizers was found be to be higher in our study than that observed in CATIE trial. The predictors for utilization of multiple concomitant psychotropic medications in CATIE trial were anxiety or depression, being female, and taking atypical antipsychotics. The other possible reason for usage of concomitant medications may relate to the lack of efficacy. ${ }^{24}$

Although, there was no study evaluating the effect of atypical switching to typical antipsychotics, there were several studies comparing the effect of both classes on the risk of hospitalization in schizophrenia patients. ${ }^{25-27}$ These studies revealed that atypical antipsychotic was associated with a lower or equal risk of hospitalization compared to typical antipsychotics. ${ }^{26,28}$ Also, in geriatric patients, studies showed a lower risk of hospitalization in patients using atypical antipsychotics than in patients using typical antipsychotics..$^{29,30}$

Our study addressed a key message differently from what has been quoted by the previous studies. ${ }^{31-33}$ Most studies compared the effect of antipsychotic switching from typical to atypical antipsychotics, ${ }^{34}$ in contrast, our study addressed this issue in the opposite way. Despite the question being answered differently, the risk of hospitalization in patients receiving typical antipsychotics was higher than that in patients receiving atypical antipsychotics. The increased risk of hospitalization is hypothesized to be due to disease relapse among the patients who switched to typical antipsychotics. A previous study ${ }^{34}$ indicated that atypical antipsychotics show superiority in relapse prevention compared to typical antipsychotics, which could lead to lower hospitalization rate. ${ }^{32,35}$

Our study elucidated that the total health care cost in atypical antipsychotic continuation group may be lower than that of atypical antipsychotic switching group due to a lower cost of hospitalization. This probably means that the lower cost of hospitalization in the atypical antipsychotic continuation group is not sufficient to offset higher medication cost. Our findings are consistent with a finding from a previous study that costcontainment policy focusing on reducing drug expenditures on chronically mentally ill patients results in unintended outcomes such as an increase in hospitalization and nursing home admission, and use of emergency mental health services. ${ }^{36}$
The strengths of our study should be highlighted. First, we used databases from different settings (both university and psychiatric hospitals) to increase generalizability of our findings to wider schizophrenia patients seen in the mental health system in Thailand. Second, we measured outcomes longitudinally using 2-year follow-up period to capture outcomes in a natural course of schizophrenia as chronic mental illness since hospitalization is a proxy of relapse of disease. ${ }^{37}$ Third, we used a state-of-art approach such as propensity score method to minimize residual confounding in our adjusted and matched analyses. The use of propensity score matching can help achieve balance between the covariates of the two groups. Therefore, the findings can be considered to mimic the outcomes observed by randomized control trials (RCTs). ${ }^{38}$

Several limitations deserve discussion. First, according to a limitation of the hospital databases, information on reasons for antipsychotic switching, disease severity, and some socioeconomic information such as educational level and patient income were not available. Second, we did not take into account patient's adherence to antipsychotics, which was considered an important factor affecting hospitalization. It was clearly shown that partial nonadherence was directly associated with an increased hospitalization. ${ }^{39}$ Third, because of the retrospective nature of the database analysis, our outcomes might not be completely captured as patients might be hospitalized in other health care facilities. Our findings might be underestimated in terms of the rate of hospitalization. Fourth, we could not separate cost analysis between generic and brand antipsychotics, and this may affect the total health care cost and medication cost because of the higher price of branded atypical antipsychotics.

Our findings have both clinical and policy implications that should be considered. Our study showed that switching from atypical antipsychotic to typical antipsychotic significantly increased the risk of hospitalization, approximately two times, without a difference in the total health care cost. These findings may caution clinicians to reconsider adverse effects of switching from atypical to typical antipsychotics, before switching the antipsychotics, in clinical practice and policy makers to carefully consider the potential impact of atypical to typical antipsychotic switching policy program before implementing the policy program at the national level.

\section{Conclusion}

In this study, we found an increased risk and rate of hospitalizations and increased health care resource utilization 
in patients who switched from atypical antipsychotics to typical antipsychotics. However, health care cost was not significantly different between the two groups. Policy makers could potentially adapt these findings in developing prospective policies and practice guidelines for treatment in schizophrenia where resources are limited to achieve both clinical efficacy and cost-containment outcomes.

\section{Acknowledgment}

The authors are pleased to acknowledge Dr Thanarat Suansanae (Faculty of Pharmacy, Mahidol University) and Dr Aporanee Chaiyakum (Faculty of Pharmaceutical Sciences, Khon Kaen University), who provided general advice and guidance in this study.

\section{Disclosure}

The authors report no conflicts of interest in this work.

\section{References}

1. Bowie CR, Depp C, McGrath JA, et al. Prediction of real-world functional disability in chronic mental disorders: a comparison of schizophrenia and bipolar disorder. Am J Psychiatry. 2010;167(9):1116-1124.

2. Freedman R. Schizophrenia. N Engl J Med. 2003;349(18): 1738-1749.

3. World Health Organization. Global Burden of Disease: 2004 Update. Geneva: WHO Press; 2008.

4. Millier A, Schmidt U, Angermeyer MC, et al. Humanistic burden in schizophrenia: a literature review. J Psychiatr Res. 2014;54:85-93.

5. Wu EQ, Birnbaum HG, Shi L, et al. The economic burden of schizophrenia in the United States in 2002. J Clin Psychiatry. 2005;66(9):1122-1129.

6. Holla B, Thirthalli J. Course and outcome of schizophrenia in asian countries: review of research in the past three decades. Asian J Psychiatr. 2015;14:3-12.

7. Tandon R. Antipsychotics in the treatment of schizophrenia: an overview. J Clin Psychiatry. 2011;72 (Suppl 1):4-8.

8. Lehman AF, Lieberman JA, Dixon LB, et al. Practice guideline for the treatment of patients with schizophrenia, second edition. Am J Psychiatry. 2004;161(Suppl 2):1-56.

9. Dixon L, Perkins D, Calmes C. Guideline Watch (September 2009): Practice Guideline for the Treatment of Patients with Schizophrenia. 2009. Available from: http://psychiatryonline.org/pb/assets/raw/ sitewide/practice_guidelines/guidelines/schizophrenia-watch.pdf. Accessed September 18, 2015.

10. Kenneth O, Jobson M. The International Psychopharmacology Algorithm Project (IPAP) - schizophrenia algorithm, schizophrenia algorithm nodes. 2005. Available from: http://www.ipap.org/schiz/index.php. Accessed September 18, 2015.

11. Moore TA, Buchanan RW, Buckley PF, et al. The Texas Medication Algorithm Project antipsychotic algorithm for schizophrenia: 2006 update. J Clin Psychiatry. 2007;68(11):1751-1762.

12. Davis JM, Chen N, Glick ID. A meta-analysis of the efficacy of second-generation antipsychotics. Arch Gen Psychiatry. 2003;60(6): 553-564.

13. Zhang JP, Gallego JA, Robinson DG, Malhotra AK, Kane JM, Correll CU. Efficacy and safety of individual second-generation vs. first-generation antipsychotics in first-episode psychosis: a systematic review and meta-analysis. Int $J$ Neuropsychopharmacol. 2013;16(6): 1205-1218.
14. Crossley NA, Constante M, McGuire P, Power P. Efficacy of atypical v. typical antipsychotics in the treatment of early psychosis: meta-analysis. Br J Psychiatry. 2010;196(6):434-439.

15. The Royal College of Psychiatrists of Thailand. Recommendations for the pharmacotherapy of mental disorders (2013). J Psychiatr Assoc Thailand. 2013;58(3):12.

16. National Drug Committee. Thailand National List of Essential Medicines 2012. Bangkok: Leligious affair publishing house; 2012.

17. Stabile M, Thomson S, Allin S, et al. Health care cost containment strategies used in four other high-income countries hold lessons for the United States. Health Aff (Millwood). 2013;32(4):643-652.

18. Rawabdeh AA. Health care cost containment strategies: the Jordanian experience. Int J Health Plann Manage. 2005;20(1):53-66.

19. Tangcharoensathien V, Tantivess S, Teerawattananon Y, Auamkul N, Jongudoumsuk P. Universal coverage and its impact on reproductive health services in Thailand. Reprod Health Matters. 2002;10(20):59-69.

20. Ministry Of Public Health Network of Unit Cost. Unit Cost Manual for Hospital. Nonthaburi: MUCC; 2011.

21. Bureau of Trade and Economic indices Ministry of Commerce Thailand. Report for Customer Price Index of Thailand Year 2002-2015 (BASE YEAR 2011). Available from: http://www.indexpr.moc.go.th/ price_present/TableIndexG_region.asp?nyear $=2558 \&$ Province_ $\operatorname{code}=5 \&$ table_name $=$ cpig_index_country\&type_code $=\mathrm{g} \&$ check $\mathrm{f}=\mathrm{i} \&$ comm_code $=4 \&$ Submit $=+\% \mathrm{~B} 5 \% \mathrm{~A} 1 \% \mathrm{C} 5 \% \mathrm{~A} 7+$. Accessed September 10, 2015.

22. Bank of Thailand. Report for exchage rate in Thailand. Available from: http://www2.bot.or.th/statistics/ReportPage.aspx? reportID=123\&language=th. Accessed September 10, 2015.

23. Chakos MH, Glick ID, Miller AL, et al. Baseline use of concomitant psychotropic medications to treat schizophrenia in the CATIE trial. Psychiatr Serv. 2006;57(8):1094-1101.

24. Hugenholtz GW, Heerdink ER, Meijer WE, Stolker JJ, Egberts AC, Nolen WA. Reasons for switching between antipsychotics in daily clinical practice. Pharmacopsychiatry. 2005;38(3):122-124.

25. Gianfrancesco F, Rajagopalan K, Wang RH. Hospitalization risks in the treatment of schizophrenia: comparison of antipsychotic medications. J Clin Psychopharmacol. 2006;26(4):401-404.

26. Kennedy J, Tien YY, Cohen LJ, et al. The association between class of antipsychotic and rates of hospitalization: results of a retrospective analysis of data from the 2005 Medicare current beneficiary survey. Clin Ther. 2009;31(12):2931-2939.

27. Lang K, Meyers JL, Korn JR, et al. Medication adherence and hospitalization among patients with schizophrenia treated with antipsychotics. Psychiatr Serv. 2010;61(12):1239-1247.

28. Al-Zakwani IS, Barron JJ, Bullano MF, Arcona S, Drury CJ, Cockerham TR. Analysis of healthcare utilization patterns and adherence in patients receiving typical and atypical antipsychotic medications. Curr Med Res Opin. 2003;19(7):619-626.

29. Aparasu RR, Jano E, Johnson ML, Chen H. Hospitalization risk associated with typical and atypical antipsychotic use in community-dwelling elderly patients. Am J Geriatr Pharmacother. 2008;6(4):198-204.

30. Aparasu RR, Chatterjee S, Chen H. Risk of hospitalization and use of first- versus second-generation antipsychotics among nursing home residents. Psychiatr Serv. 2014;65(6):781-788.

31. Voruganti L, Cortese L, Owyeumi L, et al. Switching from conventional to novel antipsychotic drugs: results of a prospective naturalistic study. Schizophr Res. 2002;57(2-3):201-208.

32. Casey DE, Carson WH, Saha AR, et al. Switching patients to aripiprazole from other antipsychotic agents: a multicenter randomized study. Psychopharmacology (Berl). 2003;166(4):391-399.

33. Lin HC, Chong MY, LeeY, Yeh WC, Lin PY. Switching of antipsychotics to aripiprazole in the treatment of schizophrenia. Chang Gung Med J. 2009;32(4):409-416.

34. Kishimoto T, Agarwal V, Kishi T, Leucht S, Kane JM, Correll CU. Relapse prevention in schizophrenia: a systematic review and metaanalysis of second-generation antipsychotics versus first-generation antipsychotics. Mol Psychiatry. 2013;18(1):53-66. 
35. Olivares JM, Sermon J, Hemels M, Schreiner A. Definitions and drivers of relapse in patients with schizophrenia: a systematic literature review. Ann Gen Psychiatry. 2013;12(1):32.

36. Soumerai S. Unintended outcomes of medicaid drug cost-containment policies on the chronically mentally ill. J Clin Psychiatry. 2003; 64(Suppl 17):19-22.

37. Burns T. Hospitalisation as an outcome measure in schizophrenia. Br J Psychiatry Suppl. 2007;50:s37-s41.
38. Austin PC. An introduction to propensity score methods for reducing the effects of confounding in observational studies. Multivariate Behav Res. 2011;46(3):399-424.

39. Kozma CM, Weiden PJ. Partial compliance with antipsychotics increases mental health hospitalizations in schizophrenic patients: analysis of a national managed care database. Am Health Drug Benefits. 2009;2(1):31-38.

\section{Publish your work in this journal}

ClinicoEconomics \& Outcomes Research is an international, peerreviewed open-access journal focusing on Health Technology Assessment, Pharmacoeconomics and Outcomes Research in the areas of diagnosis, medical devices, and clinical, surgical and pharmacological intervention. The economic impact of health policy and health systems organization also constitute important areas of coverage. The manuscript management system is completely online and includes a very quick and fair peer-review system, which is all easy to use. Visit http://www.dovepress.com/testimonials.php to read real quotes from published authors.

Submit your manuscript here: http://www.dovepress.com/clinicoeconomics-and-outcomes-research-journal 